\title{
Serviço de Atendimento Móvel de Urgência: perfil dos atendimentos de crianças e
} adolescentes

Mobile Emergency Care Service: profile of care provided to children and adolescents Servicio móvil de atención de emergencia: perfil de atención brindada a niños y adolescentes

Recebido: 18/05/2020 | Revisado: 19/05/2020 | Aceito: 22/05/2020 | Publicado: 24/05/2020

Bianca Machado Cruz Shibukawa ORCID: https://orcid.org/0000-0002-7739-7881

Universidade Estadual de Maringá, Brasil E-mail: bih.cruuz@gmail.com

Gabrieli Patricio Rissi ORCID: https://orcid.org/0000-0002-1702-4004 Universidade Estadual de Maringá, Brasil E-mail: gabrielirissi@gmail.com Marina Bennemann de Moura ORCID: https://orcid.org/0000-0001-6522-6076 Prefeitura Municipal de Maringá, Brasil E-mail: marinabenn@hotmail.com Vanessa Denardi Antoniassi Baldissera ORCID: https://orcid.org/0000-0003-1680-9165 Universidade Estadual de Maringá, Brasil E-mail: vanessadenardi@hotmail.com Ieda Harumi Higarashi ORCID: https://orcid.org/0000-0002-4205-6841 Universidade Estadual de Maringá, Brasil E-mail: ieda1618@gmail.com Maria de Fátima Garcia Lopes Merino ORCID: https://orcid.org/0000-0002-5392-1001

Universidade Estadual de Maringá, Brasil E-mail: fatimamerino@gmail.com 


\title{
Resumo
}

Objetivo: identificar o perfil de atendimentos do Serviço de Atendimento Móvel de Urgências, a crianças e adolescentes no noroeste do Paraná. Metodologia: desenvolveu-se estudo transversal de caráter quantitativo, no noroeste do Estado do Paraná. A amostra foi composta por todos os registros da equipe de atendimento do Serviço de Atendimento Móvel de Urgências. A coleta de dados ocorreu no primeiro semestre de 2017, foram extraídos dados dos atendimentos a pacientes com idade entre 0 e 19 anos. A análise dos fatores associados foi realizada por meio do cálculo da razão de chances. Resultados: Analisou-se 1483 atendimentos, dos quais 62,2\% eram adolescentes, seguido do grupo etário de lactentes e crianças, ambos representando 14,1\%. Os chamados versaram sobre transferências, problemas respiratórios, acidentes domésticos em sua grande maioria, e o local mais frequente dos chamamentos foram os domicílios. Conclusão: é necessário o fortalecimento das instituições de ensino e de saúde, para que estejam preparados para receber esta clientela, bem como a importância da difusão do funcionamento do Serviço de Atendimento Móvel de Urgências, para que este não seja utilizado apenas como um serviço de transferência.

Palavras-chave: Serviços médicos de emergência; Saúde da criança; Saúde do adolescente.

\begin{abstract}
Objective: identify the profile of care provided by the Mobile Emergency Care Service to children and adolescents in northwest Paraná. Methodology: a cross-sectional quantitative study was developed in the northwest of the State of Paraná. The sample consisted of all the records of the service team of the Mobile Emergency Service. Data collection took place in the first semester of 2017, data were extracted from the care provided to patients aged between 0 and 19 years. The analysis of associated factors was performed by calculating the odds ratio. Results: 1483 consultations were analyzed, of which $62.2 \%$ were adolescents, followed by the age group of infants and children, both representing $14.1 \%$. Conclusion: The calls dealt with transfers, breathing problems, domestic accidents in the vast majority, and the most frequent place of calls was the homes. It was concluded that the strengthening of educational and health institutions is necessary, so that they are prepared to receive this clientele, as well as the importance of spreading the functioning of the Mobile Emergency Care Service, so that it is not used only as a transfer service.
\end{abstract}

Keywords: Emergency medical services; Child health; Adolescent health.

\section{Resumen}

Objetivo: identificar el perfil de atención brindada por el Servicio Móvil de Atención de Emergencia a niños y adolescentes en el noroeste de Paraná. Metodología: estudio cuantitativo transversal en el 
noroeste del Estado de Paraná. La muestra consistió en todos los registros del equipo de servicio del Servicio de Emergencia Móvil. La recopilación de datos tuvo lugar en el primer semestre de 2017, los datos se extrajeron de la atención brindada a pacientes de entre 0 y 19 años. El análisis de los factores asociados se realizó mediante el cálculo de la odds ratio. Resultados: Se analizaron 1.483 consultas, de las cuales el 62,2\% eran adolescentes, seguidas del grupo de edad de lactantes y niños, ambos representando el 14,1\%. Las llamadas se referían a transferencias, problemas respiratorios, accidentes domésticos en la gran mayoría, y el lugar más frecuente de llamadas eran los hogares. Conclusión: el fortalecimiento de las instituciones educativas y de salud es necesario, para que estén preparadas para recibir esta clientela, así como la importancia de difundir el funcionamiento del Servicio Móvil de Atención de Emergencia, para que no se utilice solo como un servicio de transferencia.

Palabras clave: Servicios médicos de urgencia; Salud del niño; Salud del adolescente.

\section{Introdução}

A curiosidade, a imaturidade dos instintos de fuga e a sensação de invencibilidade, fazem das crianças e dos adolescentes alvos fáceis para acidentes e até mesmo práticas violentas. No Brasil de 2000 a 2010 houve um aumento de 61,4\% das taxas de mortalidade em crianças menores de um ano por causas externas e no mesmo período, um aumento de 50\% na faixa etária superior a 14 anos (Oliveira, et al., 2018, Silva, et al., 2017, Sabino, et al., 2017).

$\mathrm{O}$ atendimento pré-hospitalar adequado é o fator determinante entre a vida e a morte das vítimas, portanto com o intuito de organizar e sistematizar as respostas rápidas aos serviços de urgências e emergências, o governo lançou uma proposta de Serviços de Atendimentos Móveis de Urgência (SAMU) para antecipar o socorro à vítima, assegurando maior chance de sobrevida aos usuários atendidos (Tibães, et al., 2018).

Nesse sentido, o SAMU se constitui como o principal componente da rede de urgência e emergência pré-hospitalar, objetivando realizar o primeiro atendimento em lugares públicos que não possuem suporte para assistir pessoas com a saúde comprometida. Logo, a assistência prestada por estes serviços precisa ser qualificada, resolutiva e segura, tanto para o usuário do serviço de saúde quanto para o profissional prestador de cuidado (Orsolin, 2019).

Para orquestrar todos os desdobramentos deste serviço o SAMU foi integrado a um sistema regionalizado, hierarquizado e regulado, garantindo unidades próximas às áreas de abrangência, a fim de otimizar o atendimento e a vinculação às instituições hospitalares referenciadas (Tibães, et al., 2018, Santana, et al., 2016). 
A assistência a pacientes em situações de emergência demanda acentuado preparo técnico-científico, além de exigir considerável controle emocional dos profissionais de saúde, especialmente quando se trata da população pediátrica, a qual requer uma grande variedade de intervenções a fim de garantir as manobras de estabilidade da vida (Ribeiro, et al., 2019).

Portanto, conhecer as características dos serviços permite ao profissional antecipar as demandas, de modo que possibilite planejar as escalas profissionais e direcionar treinamento para determinado tipo de atendimento. O planejamento e o treinamento das equipes também são determinantes no sucesso ou fracasso do atendimento pré-hospitalar, portanto faz-se necessário uma análise minuciosa do território a ser coberto (Santana, et al., 2016, Silva \& Invenção, 2018).

Tendo em vista a necessidade do planejamento e domínio do serviço móvel de urgência e emergência é que se objetivou identificar o perfil de atendimentos do SAMU a crianças e adolescentes no noroeste do Paraná.

\section{Metodologia}

Estudo transversal de caráter quantitativo, realizado em um Serviço de Atendimento Móvel de Urgências (SAMU) do noroeste do Estado do Paraná que realiza atendimentos na cidade sede e mais dois municípios circunvizinhos. A amostra foi composta por todos os registros da equipe de atendimento do SAMU do ano de 2016.

Para a coleta de dados que ocorreu no primeiro semestre de 2017, agendou-se previamente com o SAMU os melhores dias e horário para a extração de dados. Os registros deste serviço eram organizados em planilhas eletrônicas por dia de atendimento.

Para organizar a extração dos dados, as pesquisadoras utilizaram um questionário estruturado de autoria própria, contendo as seguintes variáveis: município de atendimento, sexo (masculino e feminino), idade (lactente, pré-escolares, criança e adolescentes), período do atendimento (período escolar e período de férias - foi considerado período de férias os meses de janeiro, fevereiro, julho e dezembro), local da ocorrência (domicílio, via pública, estabelecimentos, hospitais e outros), e motivo do atendimento.

Após a coleta os dados as informações foram transcritas para planilhas do Programa Microsoft Excel e organizados conforme as variáveis da coletadas no instrumento estruturado. Realizou-se inicialmente análise estatística descritiva por meio de frequências, médias e porcentagens, as quais foram aplicadas por grupos etários. 
Research, Society and Development, v. 9, n. 7, e505974666, 2020

(CC BY 4.0) | ISSN 2525-3409 | DOI: http://dx.doi.org/10.33448/rsd-v9i7.4666

A análise dos fatores associados foi realizada por meio do cálculo da razão de chances (Odds Ratio - OR), com intervalo de confiança (IC) de 95\%, com os dados agrupados por faixa etária e período de atendimento. As análises foram realizadas por meio do software R, versão 3.6.3.

Para a realização da presente pesquisa, foram seguidos todos os preceitos éticos e legais contidos nas normas regulamentadoras determinadas pelas Resoluções 466/2012, 510/2016 do Conselho Nacional de Saúde, e que estabelecem as diretrizes éticas para a realização de estudos com seres humanos.

O estudo obteve o parecer de apreciação ética pelo Comitê Permanente de Ética em Pesquisa Envolvendo Seres Humanos da Universidade Estadual de Maringá sob o registro ${ }^{\circ}$ 1.196 .874$.

\section{Resultados}

No ano de 2016 o SAMU da região em questão realizou 1483 atendimentos a crianças e adolescentes de 0 a 19 anos, sendo destes atendimentos, 96,63\% foram para atender o município sede e seus distritos, sendo apenas 3,37\% dos atendimentos realizados nos municípios circunvizinhos.

Em relação ao grupo etário das crianças e adolescentes atendidos, 62,2\% (923) encontram-se na adolescência, seguido do grupo etário de lactentes e crianças, ambos representando 14,1\% (209), e por fim o grupo de pré-escolares representou 9,6\% (142) dos atendimentos. Na Tabela 1, é possível identificar o atendimento por grupo etário e sexo. 
Research, Society and Development, v. 9, n. 7, e505974666, 2020

(CC BY 4.0) | ISSN 2525-3409 | DOI: http://dx.doi.org/10.33448/rsd-v9i7.4666

Tabela 1. Distribuição de atendimentos do Serviço de Atendimento Móvel de Urgência por sexo e grupo etário. Maringá, Paraná, Brasil, 2020.

\begin{tabular}{lccccc}
\hline & \multicolumn{2}{c}{ Masculino } & \multicolumn{2}{c}{ Feminino } & $\begin{array}{c}\text { Em } \\
\text { branco }\end{array}$ \\
\cline { 2 - 6 } Grupo etário & $\mathrm{N}$ & $\%$ & $\mathrm{~N}$ & $\%$ & $\mathrm{~N}$ \\
\hline 0 a 23 meses & 112 & 53,6 & 97 & 46,4 & - \\
2 a 5 anos & 104 & 73,2 & 38 & 26,8 & - \\
6 a 12 anos & 128 & 61,2 & 81 & 38,8 & - \\
$>12$ anos & 313 & 43,4 & 408 & 56,6 & 202 \\
\hline
\end{tabular}

Fonte: Autoria própria, 2020.

Na Tabela 1 é possível identificar ainda que 13,6\% (202) dos atendimentos não havia o preenchimento da variável sexo, apresentando apenas a idade do paciente atendido. Em relação aos atendimentos segundo sexo, os meninos apresentaram maiores taxas de atendimento em todos os grupos etários, exceto na adolescência.

Os motivos para solicitar o atendimento do Serviço de Atendimento Móvel de Urgência foram diversos, sendo que as características dos chamados variaram conforme o grupo etário, conforme segue na Figura 1. 
(CC BY 4.0) | ISSN 2525-3409 | DOI: http://dx.doi.org/10.33448/rsd-v9i7.4666

Figura 1. Taxa de motivos de atendimentos realizados pelo Serviço de Atendimento Móvel de Urgência, por grupo etário. Maringá, Paraná, Brasil, 2020.

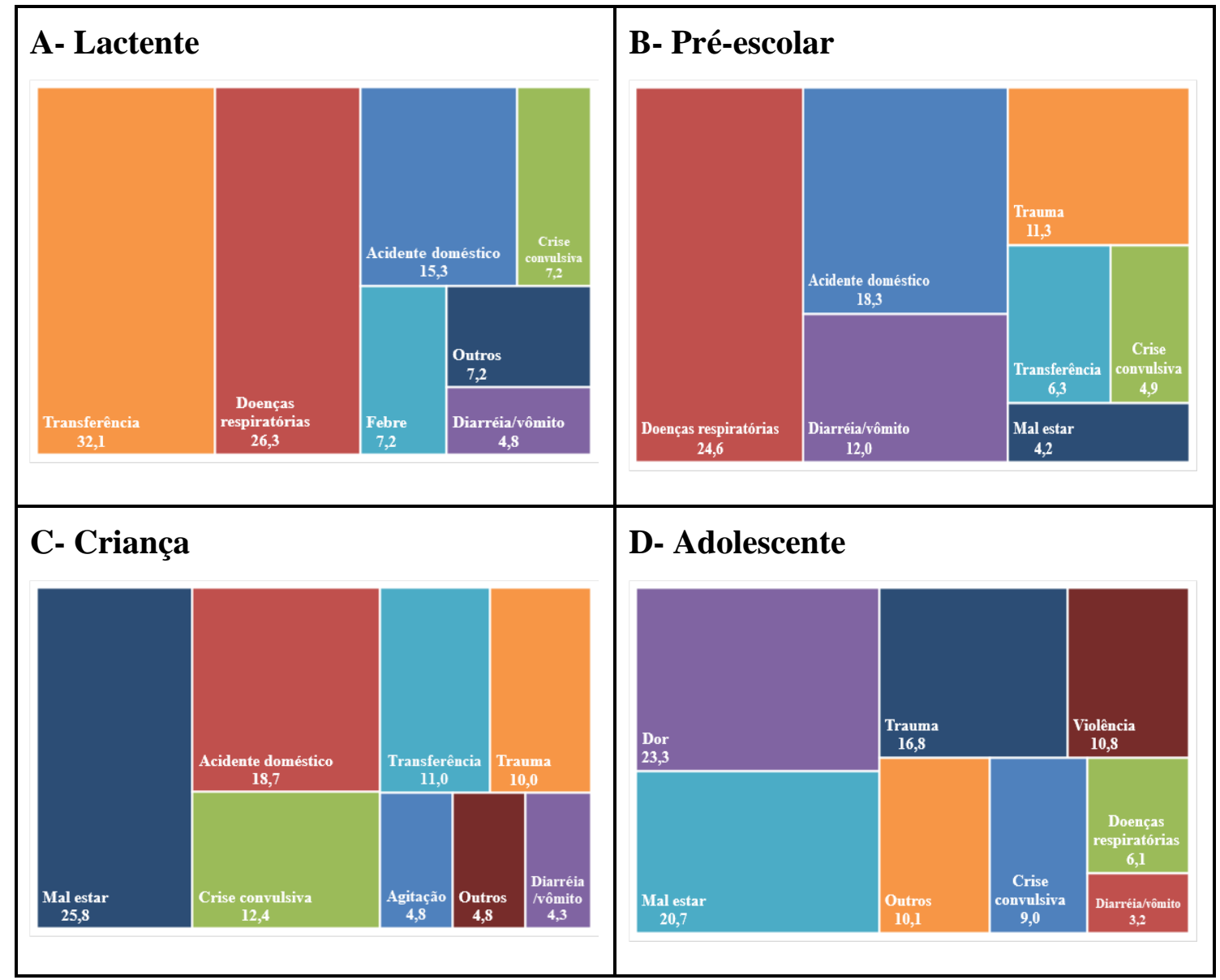

Fonte: Autoria própria, 2020.

O grupo de lactentes apresentaram mais chamados relacionados a transferência e problemas do trato respiratório, enquanto pré-escolares apresentaram mais chamados para problemas do trato respiratório e acidentes domésticos. As crianças apresentaram mais chamados por mal-estar, e também em segundo motivo os acidentes domésticos, já os adolescentes tiveram mais queixas registradas por dor e mal-estar.

Os meses com maiores atendimentos foram os de período escolares, nos quais ocorreram em média 130 chamados por mês, enquanto no período de férias, a média de atendimentos por mês foi de 111 .

À luz da análise de associação, observou-se que adolescentes têm menos chances de solicitar atendimento em período escolar, visto que este se apresentou como fator de proteção, enquanto o grupo pré-escolar demonstrou que neste período, possuem $70 \%$ mais chances de realizar um chamado ao SAMU, como segue na Tabela 2. 
(CC BY 4.0) | ISSN 2525-3409 | DOI: http://dx.doi.org/10.33448/rsd-v9i7.4666

Tabela 2. Associação de chamadas ao Serviço de Atendimento Móvel de Urgência em período escolar com os grupos etários. Maringá, Paraná, Brasil, 2020.

\begin{tabular}{lcccc}
\hline & \multicolumn{4}{c}{ Período escolar } \\
\cline { 2 - 5 } & $\mathbf{N}$ & $\mathbf{\%}$ & OR & p-valor \\
\hline Grupos etários & 143 & 9,6 & 1,1 & 0,55 \\
Lactente & 108 & 7,3 & 1,7 & $0,01^{*}$ \\
Pré-escolar & 147 & 9,9 & 1,2 & 0,21 \\
Criança & 590 & 39,8 & 0,7 & $0,04^{*}$ \\
Adolescente & & &
\end{tabular}

Fonte: Autoria própria, 2020. *Nível de confiança de $95 \%$.

Com relação a presença de acompanhante durante a assistência do SAMU, 63,9\% dos pacientes não tinham acompanhante, destes o grupo etário com maior ausência de acompanhamento, foram os adolescentes.

Sobre o local de ocorrência, o domicílio foi o gerador de maiores números de chamados para o SAMU em todos os grupos etários, exceto pelos lactentes, os quais possuíam maiores chamados dos serviços de saúde. Segue abaixo a Figura 2 com os locais de ocorrência por grupo etário. 
(CC BY 4.0) | ISSN 2525-3409 | DOI: http://dx.doi.org/10.33448/rsd-v9i7.4666

Figura 2. Taxa do local de origem do chamado para o SAMU, por grupo etário. Maringá, Paraná, Brasil, 2020.

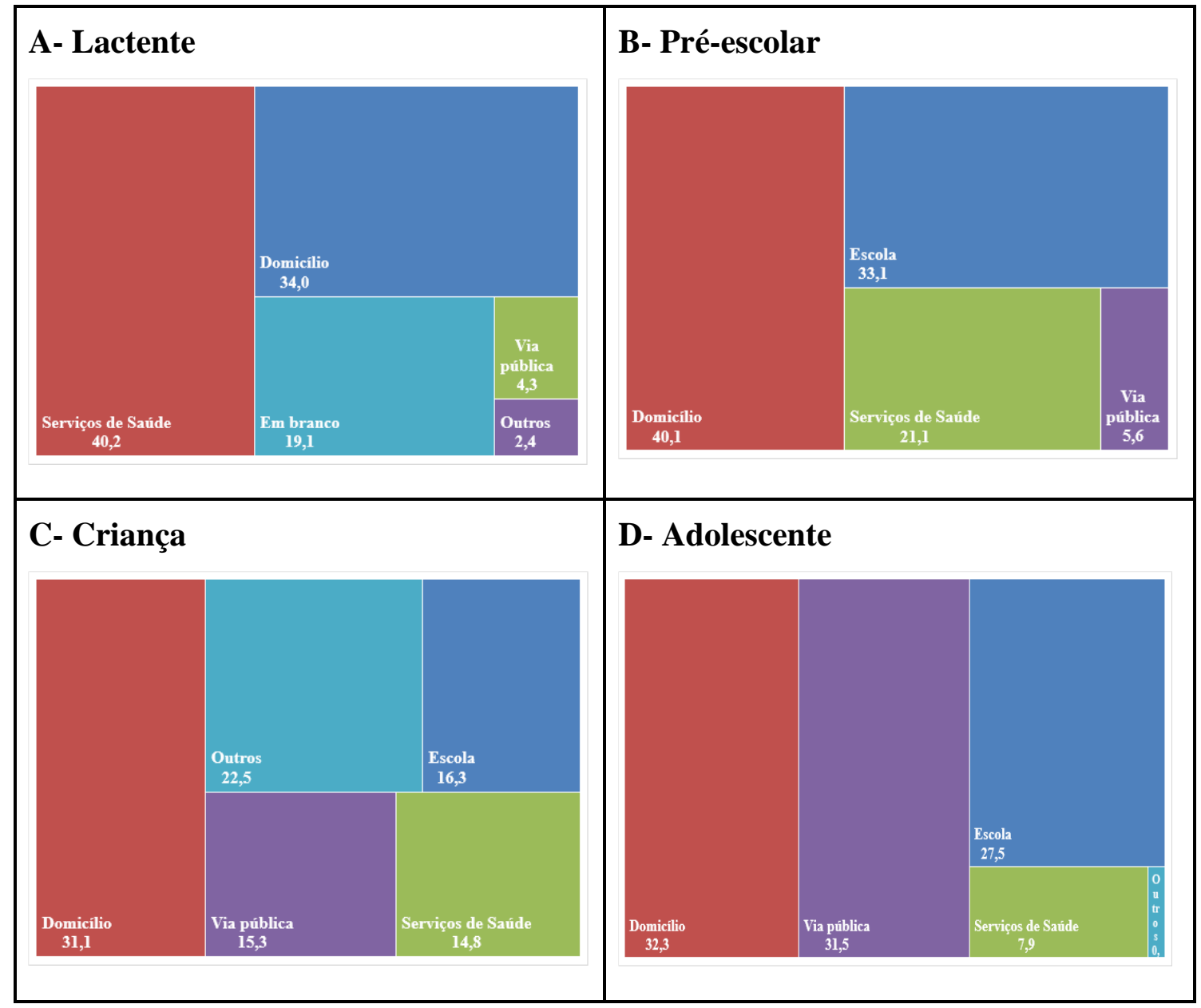

Fonte: Autoria própria, 2020.

Outros locais que também foram origem de chamados frequentes para o SAMU foram escola, serviços de saúde e via pública. Ressalta-se ainda que a via pública aumentou a quantidade de chamadas com o aumento da idade dos grupos etários, e os serviços de saúde estiveram presentes em todos os grupos etários mesmo com diferentes proporções.

\section{Discussão}

O SAMU é a principal porta de entrada para o serviço de atendimento pré-hospitalar, responsável pelo atendimento rápido, efetivo e eficaz. A equipe de trabalho é formada por médicos, enfermeiros, técnicos e condutores, os quais atendem toda a população da cidade e regiões circunvizinhas quando necessário, de acordo com o sistema de regulação do serviço (Almeida et al., 2016). 
O total de atendimentos entre crianças e adolescentes encontrados neste estudo, se aproximam ao total de atendimento encontrados em estudo realizado no Canadá, país que possui sistema de atendimento público semelhante ao do Brasil (Riney et al., 2018).

Do total de atendimentos abordados neste estudo, o grupo etário de adolescentes se destacou. Estudo realizado em São Paulo com o atendimento do SAMU, também evidenciou que este grupo etário demanda muitos atendimentos. $\mathrm{O}$ atendimento a esta parcela da população é essencial visto que possuem a possibilidade da inserção no mercado de trabalho (Almeida et al., 2016).

As crianças e lactentes possuem atendimento mais voltado para a clínica, com motivos de atendimento voltado para situações que são passíveis de serem atendidas na atenção primária em saúde, como, icterícia, otalgia entre outros. Evidenciando a necessidade da disseminação das funções fundamentais do SAMU para a população, estimulando a procura consciente, auxiliando a otimizar o funcionamento da regulação do serviço (Amaral et al., 2018).

Os resultados evidenciaram que o sexo masculino tem se destacado entre os atendimentos, exceto no caso de adolescentes, fato que coaduna com um estudo realizado nos Estados Unidos da América (EUA), no qual o sexo masculino representou 56,0\% dos atendimentos pelo serviço médico móvel (Riney, et al., 2018).

No que tange aos motivos de atendimentos, verificou-se que houve grande variação de acordo com a faixa etária. Nos lactentes, o principal motivo consistiu em transferência de serviços, seguido por doenças respiratórias e acidentes domésticos.

Torna-se válido destacar que, muitas vezes, os usuários dos serviços de saúde têm visto o SAMU como meio de transporte para uma unidade de emergência para receber o atendimento, entretanto, cabe ressaltar que essa não é a verdadeira atribuição do serviço móvel de urgência e emergência (Almeida et al., 2016). Ademais, a utilização incorreta deste serviço pode ocasionar perdas irreparáveis, especialmente quando se trata de um serviço que presta o primeiro atendimento, o qual faz grande diferença na taxa de sobrevida dos usuários em situação crítica.

Em relação aos demais motivos de atendimentos nos lactentes e àqueles apresentados pelos pré-escolares, verificou-se que houve semelhança com os achados de um estudo realizado com 239.243 crianças, o qual também apresentou porcentagens consideráveis de atendimentos por doenças respiratórias e traumas em menores de cinco anos (Silva, et al., 2019). 
Observou-se que os acidentes domésticos estiveram presentes em grande escala nos lactentes, pré-escolares e crianças, correspondendo a 15,3\%, 18,3\% e 18,7\% respectivamente. Sabe-se que o domicílio se constitui como o local no qual as crianças passam a maior parte do tempo, o que exige a supervisão e o cuidado de um adulto, especialmente considerando que as crianças possuem como características de sua personalidade a curiosidade, a qual quando associada à imaturidade física e cognitiva, não é hábil para discernir situações perigosas (Gurgel \& Monteiro, 2016).

Os resultados permitiram verificar que mal-estar, crise convulsiva e trauma constituíram-se como motivos consideráveis de atendimentos pelo serviço móvel de urgência e emergência nos grupos etários de crianças e adolescentes. Sobre isso, evidencia-se que os resultados coadunam com o abrangente estudo realizado por Silva, et al., 2019, o qual verificou que distúrbios neurológicos e traumas foram os principais motivos de atendimento pelo serviço móvel de urgência e emergência.

Uma característica observada nos motivos de atendimentos dos adolescentes foi o chamamento devido a situações de violência, correspondendo a $10,8 \%$ de todos os atendimentos da faixa etária. Um estudo realizado em âmbito nacional, o qual verificou o perfil epidemiológico dos atendimentos por violência nos serviços públicos de urgência e emergência, demonstrou preocupação com esse público alvo, pois o mesmo apresentou porcentagens elevadas de atendimentos por violência, especialmente sobre a violência doméstica e física, o que remete à atenção por parte da equipe de saúde para esse público vítima de violência (Souto, et al., 2017).

Em relação ao principal local de origem do SAMU, verificou-se que em lactentes correspondeu aos serviços de saúde e os demais grupos etários foram provenientes dos domicílios. As causas para o chamado nos serviços de saúde podem ser diversas, como transferência ou mesmo agravamento de casos.

No grupo etário dos lactentes os chamados em serviços de saúde foram atribuídos em um estudo realizado no Rio Grande do Sul, à falta de consultas na atenção primária em saúde, na qual mediante a busca espontânea para atendimento de urgências e falta de vagas para consultas, encaminha-os para outros serviços por meio do SAMU. Este estudo revelou ainda que os vários entrevistados viam este serviço como auxílio à população que não tinha condições financeiras de pagar táxi para o seu deslocamento (Amaral et. al, 2016).

Nos grupos etários dos pré-escolares, crianças e adolescentes o domicílio foi o local com maior porcentagem de chamamentos, sendo ainda o segundo local de chamadas dos lactentes. Fato a qual pode ser atribuído a quantidade de tempo que estes grupos ficam em 
seus domicílios, somado ao fator crescimento e desenvolvimento, na qual desde o nascimento novas habilidades são desenvolvidas por meio da curiosidade, persistência e treinamento (Tought, 2017).

Habilidades como andar, correr, brincar, desenhar, comunicar, aprender entre outras, exigem de todos os grupos etários a exposição a situações que propiciem este desenvolvimento, momento o qual muitas vezes acabam se ferindo involuntariamente, fator que já é esperado nesta fase da infância e adolescência (Damasceno et al., 2016).

No período de transição de grupo etário para adolescentes, o chamamento em via pública aumenta, uma vez que este grupo começa a alcançar a sua independência de ações, além de identificação com grupos, nos quais farão diversas atividades buscando o reconhecimento dos seus semelhantes. Esta fase, é ainda permeada de mudanças, como o ingresso no ensino médio, ensino superior e primeiro emprego, expondo-os a diversas situações que podem levar a uma situação de acidentes ou até mesmo esgotamento emocional, gerando várias chamadas ao SAMU.

\section{Considerações Finais}

O presente artigo auxiliou a desvendar as faltas de informações referentes ao atendimento de urgência e emergência em crianças e adolescentes pelo SAMU, conhecimento o qual permite que profissionais de saúde e educação profissional, entendam o perfil de chamamentos, para que possam organizar e treinar suas equipes, de modo a melhorar a assistência da recepção destes pacientes, visto que em situações de urgência e emergência, todos os segundos são importantes para a proteção da vida.

O objetivo deste estudo foi alcançado, uma vez que foi possível identificar o perfil de atendimento por grupos etários: lactentes apresentaram mais chamados relacionados a transferência; pré-escolares apresentaram mais chamados para problemas do trato respiratório; crianças apresentaram mais chamados por mal-estar, e, adolescentes tiveram mais queixas registradas por dor.

O local de chamadas mais frequentes para o SAMU foi o domicílio, seguido de serviços de saúde, evidenciando a importância da difusão de conhecimentos acerca da intenção inicial do SAMU, que vai além de apenas transportar pacientes, e sim de proteger a vida com o cuidado especializado em saúde em situações de urgência e emergência. 
Salienta-se ainda que novos estudos são encorajados na temática, visto a escassez de literatura frente a temática, com vistas a proporcionar novas perspectivas para todos os estudantes, professores e profissionais da área da saúde.

\section{Referências}

Almeida, PMV, Dell'Acqua, MCQ, Cyrino, CMS, Juliani, CMCM, Palhares, VC, \& Pavelqueires, S. (2016). Análise dos atendimentos do SAMU 192: Componente móvel da rede de atenção às urgências e emergências. Escola Anna Nery, 20(2):289-295. DOI: https://doi.org/10.5935/1414-8145.20160039

Amaral, CS, Reck, AZC, Souza, DS, Nuñez, ARG, Blatt, CR, Weis, AH \& Souza, EN. (2018). Urgency and emergency situations in primary care regulated by SAMU. Journal Health NPEPS, 3(1):241-252. DOI: http://dx.doi.org/10.30681/252610102881

Damasceno, SS, Nóbrega, VM, Coutinho, SED, Reichert, APS, Toso, BRGO \& Collet, N. (2016). Children's Health in Brazil: orienting basic network to Primary Health Care. Ciência \& Saúde Coletiva, 21(9):2961-2973. DOI: 10.1590/1413-81232015219. 25002015

Gurgel, AKC \& Monteiro, AI. (2016). Prevenção de acidentes domésticos infantis: susceptibilidade percebida pelas cuidadoras. Rev Fund Care Online, 8(4):5126-5135. DOI: http://dx.doi.org/10.9789/2175- 5361.2016.v8i4.5126-5135

Oliveira, JIQ, Omena, LRC, Bezerra, MVC, Oliveira, RFL \& França AMB. (2018). Características do atendimento pré-hospitalar em crianças politraumatizadas. Cadernos de Graduação: Ciências Biológicas e de Saúde, 4(2):53-66.

Orsolin, LL. (2019). Serviço de Atendimento Móvel de Urgência - SAMU: perfil dos atendimentos, potencialidades, fragilidades e estratégias. Dissertação de mestrado, Universidade Franciscana, Santa Maria, RS, Brasil.

Ribeiro, DR, Mesquita, NA, Nascimento, MCF \& Souza, LMC. (2019). Atendimento de enfermagem na área de urgência e emergência pediátrica. Revista Artigo.Com, 10 (2019):111. 
Riney, LC, Brokamp, C, Beck, AF, Pomerantz, WJ, Schwartz, HP \& Florin, TA. (2019). Emergency Medical Services Utilization Is Associated With Community Deprivation in Children. Prehosp Emerg Care, 23(2):225-232. DOI: https://doi.org/10.1080/10903127. 2018.1501124.

Sabino, R, Alves, F, Freire, AA, Oliveira, JM, Gonçalves, DL \& Fernandes, TS. (2017). The health profile of young male university students. Bol. Acad. Paulista de Psicologia, 37(93):353-374.

Santana, CJ, Silva, M, Oliveira, F, Coimbra, JAH, Marcon, SS \& Oliveira, MLF. (2016). Interaction of a Mobile Emergency Service with Families. J Health Sci, 18(4):235-239.

Silva, AMSM, Invenção, AS. (2018). Nurse's performance in cardiac arrest on a emergency department, 15(39):5-14.

Silva, LOJ, Anderson, JL, Bellolio, MF, Cambell, RL, Myers, LA, Luke, A, et al. (2019). Pediatric emergency medical services in privately insured patients: A 10-year national claims analysis. American Journal of Emergency Medicine, 37(2019): 1409-1415. DOI: https://doi.org/10.1016/j.ajem.2018.10.029

Silva, RA, Nery, AA, Rios, MA, Casotti, CA \& Alves, MS. (2017). Characterization of external causes in children and adolescents carried out in an emergency service. Rev enferm UFPE, 11:5156-62. DOI: https://doi.org/10.5205/1981-8963-v11i12a22505 p5156-5162-2017

Souto, RMCV, Barufaldi, LA, Nico, LS \& Freitas, MG. (2017). Epidemiological profile of care for violence in public urgency and emergency services in Brazilian capital, Viva 2014. Ciência \& Saúde Coletiva, 22(9)2811-2823. DOI: https://doi.org/10.1590/ 141381232017229.13342017

Tibães, HBB, Silva, DM, Alves, M, Penna, CMM \& Brito, MJM. (2018). Service Profile of the Mobile Emergency Care Service in The North of Minas Gerais State. Rev Fund Care Online, 10(3):675-82. 
Tought, P. (2018). How children succeed. Intrínseca: Rio de Janeiro, 2017.

\section{Porcentagem de contribuição de cada autor no manuscrito}

Bianca Machado Cruz Shibukawa - 20\%

Gabrieli Patricio Rissi - 20\%

Marina Bennemann de Moura - 15\%

Vanessa Denardi Antoniassi Baldissera - 15\%

Ieda Harumi Higarashi - 15\%

Maria de Fátima Garcia Lopes Merino - 15\% 